Canad. Math. Bull. Vol. 22 (4), 1979

\title{
ON BASIS CONSTANTS AND DUALITY IN BANACH SPACES
}

\author{
BY \\ LEONARD E. DOR ${ }^{(1,2)}$
}

\begin{abstract}
Every Banach space with a non-shrinking (unconditional) basis $\left(x_{i}\right)$ can be renormed so that the biorthogonal sequence $\left(x_{i}^{*}\right)$ has a much smaller (unconditional) basis constant than $\left(x_{i}\right)$. On the other hand, if the unconditional constant of $\left(x_{i}^{*}\right)$ is $C<2$ then the unconditional constant of $\left(x_{i}\right)$ is at most $C /(2-C)$. This estimate is sharp.
\end{abstract}

1. Let $\left(x_{i}\right)$ be a basis for a Banach space $X$. As is well known, the biorthogonal functionals $\left(x_{i}^{*}\right)$ are then a basic sequence in $X^{*}$, whose basis constant is dominated by that of $\left(x_{i}\right)$. Thus, the two constants coincide in the case when $\left(x_{i}\right)$ is shrinking, i.e. when $\left[x_{i}^{*}\right]=X^{*}$ (since the biorthogonal functionals $\left(x_{i}^{* *}\right)$ for $\left(x_{i}^{*}\right)$ may be then identified with $\left.\left(x_{i}\right)\right)$. We show here in Theorem 1 that if $\left(x_{i}\right)$ is non-shrinking, then $X$ can be renormed so that the basis constant of $\left(x_{i}^{*}\right)$ is less than 2 , while that of $\left(x_{i}\right)$ (and of any permutation of $\left.\left(x_{i}\right)\right)$ is as large as we wish. Thus, the basis $\left(x_{i}\right)$ is shrinking if and only if its basis constant equals the basis constant of its biorthogonal basis under all possible renormings of its span $X$. This result characterizes an isomorphic property of a basis by the permanence of an isometric property under all possible renormings of its span. In this sense it is analogous to the result of Davis and Johnson [2] who showed that a space $X$ is reflexive if (and only if) under every renorming $X$ is isometric to a dual space.

The first example of a basis $\left(x_{i}\right)$ whose basis constant is larger than that of $\left(x_{i}^{*}\right)$ was given by I. Singer [4]. His (and our) examples show that even if the basis constant of $\left(x_{i}^{*}\right)$ is 1 , no conclusion can be drawn about the basis constant of $\left(x_{i}\right)$.

Against this background, it is interesting to realize that if the unconditional constant of $\left(x_{i}^{*}\right)$ is $C<2$ and $\left(x_{i}\right)$ is a basis for $X$, then the unconditional constant of $\left(x_{i}\right)$ is bounded by a function of $C$. This is the content of Theorem 2

Received by the editors August 14, 1978.

AMS (MOS) subject classifications (1970).

Primary 46B10, 46B15.

Key words and phrases: Non-shrinking basis, biorthogonal functionals, basis constant, unconditional constant.

(1) This work was partly supported by NSF Grant MCS 76-07459.

(2) The author's present address: Wayne State University, Detroit, MI 48202. 
where we present a sharp estimate on the unconditional constant and the basis constant of the basis $\left(x_{i}\right)$ given the unconditional constant of $\left(x_{i}^{*}\right)$.

Before continuing, let us recall some notation (we follow [3] generally). Given a basis $\left(x_{n}\right)$, we put

$$
P_{\mathrm{A}} x=\sum_{i \in \mathrm{A}} x_{i}^{*}(x) x_{i}, \quad x \in X
$$

for every finite set $A \subseteq \mathbb{N}$, and put

$$
P_{n} x=\sum_{i=1}^{n} x_{i}^{*}(x) x_{i}, \quad x \in X
$$

for every $n \in \mathbb{N}$. The basis constant of $\left(x_{i}\right)$ is defined as $\sup _{n \in \mathbb{N}}\left\|P_{n}\right\|$. If $\left(x_{i}\right)$ is unconditional, we can introduce the unordered basis constant (sometimes called the "suppression constant") defined as $\sup _{\text {finite } A \subseteq \mathbb{N}}\left\|P_{\mathrm{A}}\right\|$, or, alternatively, as the supremum of the basis constants of all the permutations of $\left(x_{i}\right)$. Larger by a factor between 1 and 2 is the unconditional constant of $\left(x_{i}\right)$, defined as sup $\left\|T_{\varepsilon}\right\|$, with $\varepsilon$ ranging over $\{-1,1\}^{\mathbb{N}}$, where for such $\varepsilon$ we put

$$
T_{\varepsilon} x=\sum_{i=1}^{\infty} \varepsilon(i) x_{i}^{*}(x) x_{i}, \quad x \in X .
$$

A basis is called monotone (respectively unordered monotone, unconditionally monotone), if its (unordered, unconditional) basis constant equals 1. A basis is called spreading (sometimes called subsymmetric) if it is equivalent to all its subsequences. (Some authors require a subsymmetric basis to be unconditional by definition; therefore we prefer to use the term "spreading" here.)

In Theorem 1 we present the result mentioned above together with its unconditional analogue and with a refinement in a special case: If $\left(x_{i}\right)$ is a spreading, non-weakly-null basis for $X$, then $X$ can be renormed to make the basis constant of $\left(x_{i}\right)$ arbitrarily large, while making $\left(x_{i}^{*}\right)$ monotone, or even unordered monotone in the case when $\left(x_{i}\right)$ is unconditional. (In the latter case $\left(x_{i}\right)$ is necessarily equivalent to the usual $l_{1}$-basis.)

Our other result goes in the opposite direction. We show in Theorem 2 that if $\left(x_{i}\right)$ is a basis for $X$, and if the unconditional constant of $\left(x_{i}^{*}\right)$ is $C<2$, then the basis constant of $\left(x_{i}\right)$ is at most $1 /(2-C)$ and its unconditional constant at most $C /(2-C)$. In particular, if $\left(x_{i}^{*}\right)$ is unconditionally monotone, then so is $\left(x_{i}\right)$. Both of these estimates are sharp, as shown by the renormings presented in Theorem 1.

The author wishes to thank Professors R. Huff and P. Morris for bringing the problem to his attention, and for useful conversations on the topic.

2. Our first result characterizes shrinking bases in terms of renormings. The basic renorming method for dual spaces used here is known (see [2], e.g.). 
THEOREM 1. Let $\left(x_{i}\right)$ be a non-shrinking (respectively, an unconditional non-shrinking) basis for $X$.

(i) Given $0<\delta<1$ and $0<\theta<1$, there is an equivalent norm on $X$ under which the basis constant (respectively, the unconditional constant) of $\left(x_{i}^{*}\right)$ is less than $(2-\delta) / \theta$, while the basis constant of any permutation of $\left(x_{i}\right)$ is larger than $\theta / \delta$.

(ii) If, moreover, $\left(x_{i}\right)$ is spreading and is not weakly null, then for each $K<\infty$ there is an equivalent norm on $X$ under which $\left(x_{i}^{*}\right)$ is a monotone (respectively, unordered monotone) basic sequence, while the basis constant of any permutation of $\left(x_{i}\right)$ is larger than $K$.

Proof. (i). Choose $0<\delta, \theta<1$. (Typically, consider $\theta$ close to 1 ). Without loss of generality, $\left(x_{i}\right)$ is monotone (respectively unconditionally monotone). By our assumption, $\left[x_{i}^{*}\right] \neq X$, so there is $0 \neq \phi \in X^{*}$ satisfying

$$
\|\phi\| \leq d\left(\phi,\left[x_{i}^{*}\right]\right) \frac{2-\theta}{\theta} .
$$

Then for all $x^{*} \in\left[x_{i}^{*}\right]$ and all scalars $\alpha$,

$$
\begin{array}{rlr}
\left\|x^{*}\right\| & \leq\|\alpha \phi\|+\left\|x^{*}-\alpha \phi\right\| \\
& \leq \frac{2-\theta}{\theta} d\left(\alpha \phi,\left[x_{i}^{*}\right]\right)+\left\|x^{*}-\alpha \phi\right\| & \text { by (1) } \\
& \leq \frac{2}{\theta}\left\|x^{*}-\alpha \phi\right\| & \text { since } x^{*} \in\left[x_{i}^{*}\right]
\end{array}
$$

so we have, taking infimum over all $\alpha$,

$$
d\left(x^{*},[\phi]\right) \geq \frac{\theta}{2}\left\|x^{*}\right\|, \quad \text { all } x^{*} \in\left[x_{i}^{*}\right] .
$$

We define a new norm $f \rightarrow|f|$ on $X^{*}$ by

$$
|f|=(2-2 \delta) d(f,[\phi])+\delta\|f\|, \quad f \in X^{*} .
$$

We clearly have

$$
\delta\|f\| \leq|f| \leq(2-\delta)\|f\|, \quad f \in X^{*} .
$$

so $|\cdot|$ is a norm on $X^{*}$ which is equivalent to $\|\cdot\|$. Now by the Hahn-Banach theorem,

$$
d(f,[\phi])=\sup \{f(x) ; \quad\|x\| \leq 1, \phi(x)=0\}, \text { for all } f \in X^{*} .
$$

Therefore $d(\cdot,[\phi])$, and hence also $|\cdot|$, is lower-semicontinuous for the weak ${ }^{*}$ topology, and thus $|\cdot|$ induces a predual norm $|\cdot|$ on $X$, which by (4) is 
equivalent to the original one. We shall show that this norm satisfies the conclusions of (i).

First notice that for $x^{*} \in\left[x_{i}^{*}\right]$, we have, using (2) and the definition (3),

$$
\begin{aligned}
\left|x^{*}\right| & \geq(2-2 \delta) \frac{\theta}{2}\left\|x^{*}\right\|+\delta\left\|x^{*}\right\| \\
& =(\theta-\theta \delta+\delta)\left\|x^{*}\right\| \geq \theta\left\|x^{*}\right\| .
\end{aligned}
$$

This, together with (4), shows that the (unconditional) basis constant of $\left(x_{i}^{*}\right)$ in the new norm is $(2-\delta) / \theta$ at most, since the corresponding constant in the old norm was 1 .

On the other hand, $|\phi|=\delta\|\phi\|$, while $\varlimsup_{\lim _{n \rightarrow \infty}}\left|P_{n}^{*} \phi\right| \geq \theta \varlimsup_{\lim _{n \rightarrow \infty}}\left\|P_{n}^{*} \phi\right\| \geq \theta\|\phi\|$ (the first inequality holds by (6), since $P_{n}^{*} \phi \in\left[x_{i}^{*}\right]$, all $n$; the second inequality holds since $\phi=\left(w^{*}\right) \lim _{n \rightarrow \infty} P_{n}^{*}(\phi)$. Thus $\varlimsup_{\lim _{n \rightarrow \infty}}\left|P_{n}\right| \geq \varlimsup_{\lim _{n \rightarrow \infty}}\left|P_{n}^{k} \phi\right| /|\phi| \geq \theta / \delta$. This argument applies to any reordering of $\left(x_{i}\right)$, thus concluding the proof of (i).

(ii). For each strictly increasing function $\sigma: \mathbb{N} \rightarrow \mathbb{N}$ let $S_{\sigma}$ be the operator on $X$ given by $S_{\sigma}\left(\sum_{i=1}^{\infty} \alpha_{i} x_{i}\right)=\sum_{i=1}^{\infty} \alpha_{i} x_{\sigma(i)}$. It follows from our assumption on $\left(x_{i}\right)$ that $\sup \left\{\left\|S_{\sigma}\right\| ; \sigma: \mathbb{N} \rightarrow \mathbb{N}\right.$ strictly increasing $\}<\infty$.

Thus, replacing the original norm by the norm $x \rightarrow \sup _{\sigma}\left\|S_{\sigma} x\right\|$ we may assume that each $S_{\sigma}$ is a contraction of $X$ onto $\left[x_{\sigma(j)} ; j \in \mathbb{N}\right]$, and that $\left(x_{i}\right)$ is a normalized basis with basis constant 1 (respectively, unconditional constant 1 ).

ClaIM. There is a functional $\phi \in X^{*}$ satisfying $\phi\left(x_{i}\right)=1$, all $i$.

In fact, there is $\psi^{\prime} \in X^{*}$ such that $\psi^{\prime}\left(x_{i}\right) \not \rightarrow 0$. Then there are a scalar $c$ and a subsequence $\left(x_{\sigma(i)}\right)$ of $\left(x_{i}\right)$ satisfying

$$
\sum_{i=1}^{\infty}\left|c \psi^{\prime}\left(x_{\sigma(i)}\right)-1\right| \leq 1
$$

Taking $\psi=S_{\sigma}^{*}\left(c \psi^{\prime}\right)$ we see that $\sum_{i=1}^{\infty}\left|\psi\left(x_{i}\right)-1\right| \leq 1$. Now for any $n$, and any $x=\sum_{i=1}^{n} \alpha_{i} x_{i}$, we have $\left|\alpha_{i}\right| \leq 2\|x\|$, all $i \leq n$, and so $\left|\sum_{i=1}^{n} \alpha_{i}-\psi(x)\right| \leq$ $\sum_{i=1}^{n}\left|\alpha_{i}\left(1-\psi\left(x_{i}\right)\right)\right| \leq 2\|x\|$ and so the functional $\phi: \sum_{i=1}^{\infty} \alpha_{i} x_{i} \rightarrow \sum_{i=1}^{\infty} \alpha_{i}$ is well defined on $X,\|\phi\| \leq\|\psi\|+2$ and $\phi\left(x_{i}\right)=1$, all $i$, proving the Claim.

Now if $x^{*}=\sum_{i=1}^{n} \alpha_{i} x_{i}^{*}$, then for all $x \in X$,

$$
|\phi(x)|=\left|\phi\left(S_{\sigma} x\right)\right|=\left|\left(\phi-x^{*}\right)\left(S_{\sigma} x\right)\right| \leq\left\|\phi-x^{*}\right\|\|x\|,
$$

where we take $\sigma: i \rightarrow i+n$.

Consequently, $\|\phi\|=d\left(\phi,\left[x_{i}^{*}\right]\right)$, and so we have (2) with $\theta=1$. As in the proof of (i), we define an equivalent dual norm $|\cdot|$ on $X^{*}$ by (3), with $\delta<K^{-1}$. By the argument there, the basis constant of $\left(x_{i}\right)$ taken in any order is at least $\delta^{-1}>K$ under the new norm. We claim that

$$
d\left(\sum_{i=1}^{n} \alpha_{i} x_{i}^{*},[\phi]\right) \leq d\left(\sum_{i=1}^{n+1} \alpha_{i} x_{i}^{*},[\phi]\right)
$$


for all $n, \alpha_{1}, \ldots, \alpha_{n+1}$. This will show that $\left(x_{i}^{*}\right)$ is a monotone basic sequence under the new norm $|\cdot|$. To prove (7) we use (5): let $x \in X$ satisfy $\|x\|=1$ and $\phi(x)=0$ and let $\sigma(i)=i$ for $i \leq n$ and $=i+1$ for $i>n$. Then $\phi\left(S_{\sigma} x\right)=0$ also, and we have

$$
\left|\left(\sum_{i=1}^{n} \alpha_{i} x_{i}^{*}\right)(x)\right|=\left|\left(\sum_{i=1}^{n} \alpha_{i} x_{i}^{*}\right)\left(S_{\sigma} x\right)\right|=\left|\left(\sum_{i=1}^{n+1} \alpha_{i} x_{i}^{*}\right)\left(S_{\sigma} x\right)\right| \leq d\left(\sum_{i=1}^{n+1} \alpha_{i} x_{i}^{*},[\phi]\right)
$$

by (5) and since $\left\|S_{\sigma} x\right\| \leq\|x\|=1$. Applying (5) again, we obtain (7).

If $\left(x_{i}\right)$ is in addition unconditional, and was assumed to be unordered monotone, the same renorming (3) will leave $\left(x_{i}^{*}\right)$ unordered monotone, since $\phi$ is invariant under permutations of $\left(x_{i}\right)$ and therefore (7) holds for all permutations of $\left(x_{i}^{*}\right)$.

3. It is conceivable that every non-shrinking (respectively, unconditional non-shrinking) basis $\left(x_{i}\right)$ can be renormed so that $\left(x_{i}^{*}\right)$ is (unordered) monotone while $\left(x_{i}\right)$ has a large basis constant. In fact, we have shown in Theorem 1(ii) above that many of them can be so renormed. The next result shows that an analogous renorming is never possible with regard to the unconditional constant.

Theorem 2. Let $1 \leq C<2$. If $\left(x_{i}\right)$ is a basis for a Banach space $X$, and if $\left(x_{i}^{*}\right)$ has unconditional constant $C$, then

(i) The unordered basis constant of $\left(x_{i}\right)$ is at most $1 /(2-C)$

(ii) the unconditional basis constant of $\left(x_{i}\right)$ is at most $C /(2-C)$.

In particular, if $\left(x_{i}^{*}\right)$ is unconditionally monotone, then so is $\left(x_{i}\right)$.

Proof. Let $f \in X^{*}$. Then for each $m \in \mathbb{N}$,

$$
\begin{aligned}
\left\|P_{m}^{*} f\right\| & \leq \frac{1}{2}\|f\|+\frac{1}{2}\left\|P_{m}^{*} f-\left(I-P_{m}^{*}\right) f\right\| \\
& \leq \frac{1}{2}\|f\|+\frac{1}{2} \sup _{n \geq m}\left\|P_{m}^{*} f-\left(P_{n}^{*}-P_{m}^{*}\right) f\right\| \\
& \leq \frac{1}{2}\|f\|+\frac{C}{2} \sup _{n \geq m}\left\|P_{m}^{*} f+\left(P_{n}^{*}-P_{m}^{*}\right) f\right\| \\
& \leq \frac{1}{2}\|f\|+\frac{C}{2} \sup _{n}\left\|P_{n}^{*} f\right\|
\end{aligned}
$$

The second inequality holds since $g=P_{m}^{*} f-\left(I-P_{m}^{*}\right) f$ is the weak ${ }^{*}$ limit of $\left(P_{n}^{*} g\right.$ ) as $n \rightarrow \infty$, and the third one holds by the unconditionality of $\left(x_{i}^{*}\right)$. Taking supremum on the left hand side, we get that

$$
\sup _{n}\left\|P_{n}^{*} f\right\| \leq \frac{1}{2-C}\|f\|,
$$

so $\left\|P_{n}\right\| \leq 1 /(2-C)$ for all $n$, and since our assumptions are invariant under 
permutations of $\left(x_{i}\right)$, we see that the unordered basis constant of $\left(x_{i}\right)$ is at most $1 /(2-C)$, which proves (i). (ii) follows from (i) and the following simple observation: if $\left(x_{i}\right)$ is a basis for $X$ with basis constant $K$, then the natural map $S$ of $X$ into $\left[x_{i}^{*}\right]^{*}$ satisfies $\|S\| \leq 1$ and $\left\|S^{-1}\right\| \leq K$. Thus the unconditional constant of $\left(x_{i}\right)$ is at most $K$ times the unconditional constant of $\left(S x_{i}\right)$ which in turn is less than or equal the unconditional constant of $\left(x_{i}^{*}\right)$, since $\left(S x_{i}\right)$ is the biorthogonal sequence to $\left(x_{i}^{*}\right)$ in $\left[x_{i}^{*}\right]^{*}$.

Remarks. (a) To see that the constants of Theorem 2 are the best possible, consider the usual basis $\left(x_{i}\right)$ of $l_{1}$ under the renorming presented in the proof of Theorem 1(ii). Namely, the dual norm on $l_{\infty}$ is given by

$$
\left|\left(\alpha_{i}\right)\right|=\delta \sup _{i}\left|\alpha_{i}\right|+(1-\delta)\left\{\sup _{i} \alpha_{i}-\inf _{i} \alpha_{i}\right\}
$$

for all $\left(\alpha_{i}\right) \in l_{\infty}$. Then for $x^{*} \in c_{o}$ we have that $\left\|x^{*}\right\|_{\infty} \leq\left|x^{*}\right| \leq(2-\delta)\left\|x^{*}\right\|_{\infty}$, so that the unconditional constant $C$ of $\left(x_{i}^{*}\right)$ is at most $(2-\delta)$. On the other hand for $\varphi=(1,1,1, \ldots)$, we have $|\varphi|=\delta,\left|P_{1}^{*} \varphi\right|=\left|x_{1}^{*}\right|=\delta+(1-\delta)=1$, while $\left|T_{\varepsilon} \varphi\right|=$ $\delta+(2-2 \delta)=2-\delta$ for any non-trivial choice of signs. Thus the basis constant of $\left(x_{i}\right)$ is at least $1 / \delta \geq 1 /(2-C)$, while the unconditional constant of $\left(x_{i}\right)$ is at least $(2-\delta) / \delta \geq C /(2-C)$. Thus the inequalities of Theorem 2 become equalities in this case (and $C=2-\delta$ ).

(b) In Theorem 2 we assumed that $\left(x_{i}\right)$ was a basis for $X$. The following problem is open: Let $\left(x_{i}, x_{i}^{*}\right)$ be a biorthogonal system in $X \times X^{*}$. Assume that $\left[x_{i}\right]=X$ and that $\left(x_{i}^{*}\right)$ has an unconditional constant $C<2$. Is $\left(x_{i}\right)$ a (necessarily unconditional) basis for $X$ ? What if $C=1$ ? A related problem is: Let $\left(x_{1}, x_{i}^{*}\right)$ be a biorthogonal system in $X \times X^{*}$. If $\left[x_{i}\right]=X$, and if $\left(x_{i}^{*}\right)$ is a boundedly complete basic sequence, is $\left(x_{i}\right)$ a basic sequence?

It was observed by I. Singer that there is a biorthogonal system $\left(x_{i}, x_{i}^{*}\right)$ in $X \times X^{*}$ with $\left[x_{i}\right]=X$ so that $\left(x_{i}^{*}\right)$ is a basic sequence and $\left(x_{i}\right)$ is not. This follows easily from the existence of examples such as in Theorem 1(i).

(c) Let $\left(x_{i}\right)$ be a basis for $X$, and let $S$ be the natural map of $X$ into $\left[x_{i}^{*}\right]^{*}$. In his discussion of basis constants in [5] I. Singer asked the following question: Assume that the basis constants of $\left(x_{i}\right)$ and of $\left(x_{i}^{*}\right)$ are equal. Is $S$ necessarily an isometric embedding: (i.e., does it follow that $\left[x_{i}^{*}\right]$ precisely norms $X$.) It is easy to construct a counter-example: Fix $C>1$. Let $\left(y_{1}, y_{2}\right)$ be a basis of constant $C$ for the two dimensional space $Y$, and let $\left(z_{i}\right)$ be a basis for a Banach space $Z$, so that the basis constant of $\left(z_{i}\right)$ is $C$ while that of $\left(z_{i}^{*}\right)$ is 1 . Let $X=(Y \oplus Z)_{2}$, and let $\left(x_{i}\right)$ be the sequence $\left(y_{1}, y_{2}, z_{1}, z_{2}, z_{3}, \ldots\right)$ in $X$. The basis constants of $\left(x_{i}\right)$ and $\left(x_{i}^{*}\right)$ are both $C$, but $\left\|S^{-1}\right\| \geq C$.

ADDED IN PROOF: After this paper had been typeset, the author has discovered another result related to Theorem 1: If $X$ is a non-reflexive Banch space, 
and $K<\infty$, then there is a basic sequence $\left(y_{i}\right)$ in $X$ such that the basis constant of $\left(y_{i}\right)$ is larger than $K$, while the basis constant of the dual sequence $\left(y_{i}^{*}\right)$ in $\left[y_{i}\right]^{*}$ is less than 10

\section{REFERENCES}

1. W. J. Davis, D. W. Dean, B-L. Lin, Bibasic sequences and norming basic sequences, Transactions AMS 176 (1973), 89-102.

2. W. J. Davis, W. B. Johnson, A renorming of nonreflexive spaces, Proc. AMS 37 (1973), 486-488.

3. J. Lindenstrauss, L. Tzafriri, Classical Banach spaces I, Springer-Verlag, Berlin 1977.

4. I. Singer, On the constants of basic sequences in Banach spaces, Studia Math. 31 (1968), $125-134$.

5. I. Singer, Bases in Banach spaces I, Springer-Verlag, Berlin-Heidelberg, New York 1970.

DePARTMENT OF MATHEMATICS

UNIVERSTTY OF ILLINOIS

URBANA, ILLINOIS 61801 